Research Article

\title{
Preview Tracking Control of Linear Periodic Switched Systems with Dwell Time
}

\author{
Fucheng Liao $\mathbb{D}^{1},{ }^{1}$ Lijie Cui $\mathbb{D}^{1},{ }^{1}$ Yanrong Lu $\mathbb{D}^{2},{ }^{2}$ and Jiamei Deng $\mathbb{D}^{3}$ \\ ${ }^{1}$ School of Mathematics and Physics, University of Science and Technology Beijing, Beijing 100083, China \\ ${ }^{2}$ School of Automation and Electrical Engineering, University of Science and Technology Beijing, Beijing 100083, China \\ ${ }^{3}$ School of Built Environment, Engineering, and Computing, Leeds Beckett University, Leeds LS6 3QS, UK
}

Correspondence should be addressed to Fucheng Liao; fcliao@ustb.edu.cn

Received 3 May 2020; Revised 13 July 2020; Accepted 21 August 2020; Published 22 October 2020

Academic Editor: Łukasz Jankowski

Copyright $\odot 2020$ Fucheng Liao et al. This is an open access article distributed under the Creative Commons Attribution License, which permits unrestricted use, distribution, and reproduction in any medium, provided the original work is properly cited.

This paper studies the preview tracking control problem for linear discrete-time periodic switched systems. Firstly, an augmented error system is constructed for each subsystem by stabilizing the augmented error systems through the method of optimal preview control, and the tracking problem of the switched system is transformed into the switched stability problem of closed-loop augmented error systems. Secondly, a switched Lyapunov function method is applied to search the minimal dwell time satisfying the switched stability of the closed-loop augmented error systems. Thirdly, the switched preview control input is solved from the controller of the individual augmented error system, and then the sufficient conditions and the preview controller can be obtained to guarantee the solvability of the original periodic switched preview tracking problem. Finally, numerical simulations show the effectiveness of the stabilization design method.

\section{Introduction}

Switched system theories are a hot topic of control theory research in recent years [1-5]. Switched systems consist of a family of continuous-time or discrete-time subsystems and a switching rule, which defines the running time of a specific subsystem. The switching rule is always expressed by a piecewise constant function and indicates that there is one and only one subsystem working during the interval between any two switching times. The switched systems are not only widely investigated in theory but also employed in engineering practice, such as robot control [6] and communication network control $[7,8]$. Most of the theoretical results about switched systems focus on stability analysis. Stability analysis is mainly divided into two categories: stability under arbitrary switching and stability with constraints on switching. Lu and Zhao [9] gave the sufficient conditions for the asymptotical stability of switched time-delay systems and the selection method of the switching rule. In addition, in the case of constrained switching rule, Sun et al. [10] investigated the asymptotic stability of linear periodic switched systems based on the dwell-time method, which provided the sufficient conditions for the asymptotic stability of the switched systems when all of the subsystems were stable. The results were also extended to the situations under which parts of the subsystems are unstable. Hespanha [11] pointed out that when the switching rule is time-constrained, the uniform asymptotic stability and exponential stability of the system are equivalent, but the conclusion is not true for a state-dependent switching rule. Note that the dwell-time switching requires the running time interval between any two consecutive switching bigger than a constant. However, as pointed out in [12], this will lead to a certain conservativeness. To this end, Zhao et al. [12] considered the stability and stabilization problems of the switched systems using the mode-dependent average dwell-time method. As for the nonlinear switched systems, $\mathrm{Wu}$ [13] studied the feedback stabilization of arbitrary switch between two multi-input nonlinear subsystems and obtained the global stability condition for an existing state feedback controller. In recent years, with the development of computer control techniques, sampled-data control becomes more and more important. 
Li et al. [14] considered the global stabilization for a class of switched nonlinear systems and proposed a sample-data controller to guarantee the stability of closed-loop systems under an average dwell-time constraint. The same problem was also investigated in [15], where fuzzy logic systems were employed to approximate unknown nonlinear terms.

In a tracking or disturbance rejection problem, when the future information of a reference signal or external disturbance, which is also termed as preview information, is known in advance, the preview control theory will be adopted to solve the problem by constructing an augmented error system that contains the known future information. As a result, the original preview control problem can be transformed into a regulator problem [16]. Based on this idea, different branches of preview control theory have been extensively developed. Cao and Liao [17] investigated the preview control problem of linear descriptor systems with multirates. Considering the time-variable delay, Li and Liao [18] adopted a model transformation method to approximate the time-varying state delay and derived the sufficient conditions for the asymptotic stability of the closed-loop systems via the scaled small gain theorem as well as the linear matrix inequality (LMI) technique. Recently, Li and Yuan [19] extended this method to address the scenario of polytopic uncertain discrete-time systems. In fact, the preview control problem for stochastic systems is very challenging. Wang et al. [20] considered the $H_{\infty}$ disturbance preview control problem for discrete-time systems, where the projection principle in the indefinite space was adopted. It is known that a preview controller always contains two parts, namely, feedback and preview feedforward compensation. In the literature mentioned above, these two parts are designed simultaneously through optimal control. Very recently, a new LMI synthesis method was proposed in [21] to design preview feedforward for a given closed-loop system. It should also be pointed out that the regulator problem of an augmented error system is also considered as the stability problem of the closed-loop augmented error system. Therefore, based on the stability theory of switched systems, it is reasonable and feasible to study the preview control problem of the switched systems.

This paper intends to study the preview control problem of periodical switched systems. Firstly, the state augmented technique of preview control is used to construct an augmented error system for each subsystem. Then, according to the optimal control theory, the controller of the switched augmented error systems is obtained, and the sufficient condition for the global asymptotic stability of closed-loop systems is given. Finally, the optimal preview controller is designed using preview control theories. The paper has the following three contributions:

(1) This paper presents a solution to the preview tracking problem of switched systems. To avoid the difference operation for the whole switched systems and meanwhile include the previewable information in the controller, an augmented error system is constructed for each subsystem, which successfully converts the original problem into the asymptotical stability problem for the switched closed-loop augmented error subsystems.

(2) The dwell-time condition, which heavily depends on the stable coefficient matrices of the closed-loop augmented error subsystems, is obtained to ensure the asymptotical stability of the switched closed-loop augmented error systems and the solvability of the preview tracking problem for switched systems.

(3) Liao et al. [22] investigated the preview tracking problem of the periodic system. It should be pointed out that when the dwell times of each subsystem are equivalent, the periodically switched systems will be changed into the general periodic systems. As a result, the approach in this paper can provide a new insight to reconsider the issue in [22].

1.1. Notations. Throughout this paper, $A>0$ indicates that $A$ is a positive definite matrix, $\operatorname{rank}(A)$ denotes the $\operatorname{rank}$ of $A$, $x \in R^{n}$ means that $x$ is an $n$-dimensional vector, $A \in R^{n \times r}$ means that $A$ is a real matrix of $n \times r,\|x\|$ denotes the Euclid norm for vector $x,\|A\|$ denotes the matrix norm derived from the vector Euclid norm, and $\max \{a, b\}$ means the maximum value between $a$ and $b$.

\section{Problem Formulation and Basic Assumptions}

Consider the switched system as follows:

$$
\left\{\begin{array}{l}
x(k+1)=A_{\sigma(k)} x(k)+B_{\sigma(k)} u(k), \\
y(k)=C_{\sigma(k)} x(k),
\end{array}\right.
$$

where $x(k) \in R^{n}$ is the state vector, $u(k) \in R^{p}$ is the input vector, $y(k) \in R^{q}$ is the output vector, and $A_{\sigma(k)} \in R^{n \times n}$, $B_{\sigma(k)} \in R^{n \times p}$, and $C_{\sigma(k)} \in R^{q \times n}$ are known constant matrices. $\sigma(k)$ is the switching signal, which takes its values in the finite set $I=\{1, \ldots, m\} ; m>1$ denotes the number of subsystems. $\sigma(k)=i$ means that the subsystem

$$
\left\{\begin{array}{l}
x(k+1)=A_{i} x(k)+B_{i} u(k), \\
y(k)=C_{i} x(k)
\end{array}\right.
$$

is working at time $k$.

Remark 1. In fact, system (1) is a piecewise linear system. For the convenience of description, the term "subsystem" is adopted from large-scale system theories, and system (2) is referred to as the $i$ th subsystem of system (1).

In the following, the periodic switching rule will be considered. Specifically, the switching signal $\sigma(k)$ always switches from subsystem 1 to subsystem $m$ in turn repeatedly. $T$ is assumed to be the switching period; then, the switching sequence can be denoted as follows:

$$
\Sigma_{T}=\left\{x_{0} ;\left(1, k_{1}+l T\right),\left(2, k_{2}+l T\right), \ldots,\left(m, k_{m}+l T\right) \mid l=0,1,2, \ldots\right\},
$$

where $k_{1}$ is the initial time and always taken as $k_{1}=0 .\left(i, k_{i}+\right.$ $l T$ ) means that the $i$ th subsystem is activated at time $k_{i}+l T$, 
and its running time is $T_{i}=k_{i+1}-k_{i}, T_{i}>0, i=1, \ldots, m . T_{i}$ is the running time of the $i$ th subsystem with $\sum_{i=1}^{m} T_{i}=T$.

Based on subsystem (2), switching system (1) can be represented as

$$
\begin{aligned}
& \left\{\begin{array}{l}
x(k+1)=A_{i} x(k)+B_{i} u(k), \\
y(k)=C_{i} x(k),
\end{array}\right. \\
& k \in\left[l T+k_{i}, l T+k_{i+1}\right), i=1, \ldots, m, l=0,1, \ldots
\end{aligned}
$$

The reference signal is $r(k) \in R^{q}$. The purpose of this paper is to design a preview controller which makes the output $y(k)$ of system (1) track the reference signal $r(k)$ asymptotically without a static error. Therefore, the error signal is defined as

$$
e(k)=y(k)-r(k) .
$$

A quadratic performance index function with discount factor $\lambda \in(0,1)$ is introduced:

$$
J=\sum_{k=k_{1}}^{\infty}\left[\lambda^{-2 k}\left(e^{T}(k) Q_{e} e(k)+\Delta u^{T}(k) R \Delta u(k)\right)\right],
$$

where $Q_{e}>0$ and $R>0$ are weighted matrices.

Remark 2. In quadratic performance index function (6), the discount factor $\lambda$ will make the closed-loop system of the augmented error system exponentially stable [23].

The following are the basic assumptions for preview control:

A1: the matrix $\left[\begin{array}{cc}A_{i}-I & B_{i} \\ C_{i} & 0\end{array}\right]$ is of full row rank, $i=1, \ldots, m$

A2: $\left(A_{i}, B_{i}\right)$ are stabilizable, and $\left(C_{i}, A_{i}\right)$ are detectable, $i=1, \ldots, m$

A3: the reference signal $r(k)$ is previewable with preview length $M_{R}$, that is, $r(k), r(k+1), \ldots, r\left(k+M_{R}\right)$ are available at the current time $k$, and $r(k+j)=r\left(k+M_{R}\right), j=M_{R}+1, M_{R}+2, \ldots$

\section{Controller of the Error System of the Isolated Subsystem}

After constructing an augmented error system for each subsystem (2), the idea of the controller design of system (4) is illustrated in the following. Firstly, the controller is designed for each augmented error system under quadratic performance index function (6). Secondly, each augmented error system corresponds to a closed-loop system, and the preview tracking problem of switched system (4) will be transformed into the switched stability problem of these closed-loop systems. It is noted that the final state of the $i$ th closed-loop system in time interval $\left[k_{i}+l T, k_{i+1}+l T\right)$ is just the initial state of the $(i+1)$ th closed-loop system in time interval $\left[k_{i+1}+l T, k_{i+2}+l T\right)$.

The procedure in constructing the augmented error system for each subsystem (2) is given in the following. Utilizing the difference operator $\Delta$

$$
\Delta v(k)=v(k+1)-v(k)
$$

to subsystem (4) and error vector (5), we have

$$
\begin{gathered}
\Delta x(k+1)=A_{i} \Delta x(k)+B_{i} \Delta u(k), \\
\Delta e(k)=\Delta y(k)-\Delta r(k) .
\end{gathered}
$$

Substituting $\Delta e(k)=e(k+1)-e(k)$ into (9) gives

$$
e(k+1)=e(k)+C_{i} \Delta x(k)-\Delta r(k) .
$$

Combining equations (8) and (10), the primary augmented error system is obtained as follows:

$$
\widetilde{x}(k+1)=\widetilde{A}_{i} \tilde{x}(k)+\widetilde{B}_{i} \Delta u(k)+B_{R} \Delta r(k)
$$

with

$$
\begin{aligned}
\widetilde{x}(k) & =\left[\begin{array}{c}
e(k) \\
\Delta x(k)
\end{array}\right], \\
\tilde{A}_{i} & =\left[\begin{array}{ll}
I & C_{i} \\
0 & A_{i}
\end{array}\right], \\
\widetilde{B}_{i} & =\left[\begin{array}{c}
0 \\
B_{i}
\end{array}\right], \\
B_{R} & =\left[\begin{array}{c}
-I \\
0
\end{array}\right] .
\end{aligned}
$$

According to A3, because $r(k), r(k+1), \ldots, r\left(k+M_{R}\right)$ are known in advance at time $k, \Delta r(k), \Delta r(k+1), \ldots$, $\Delta r\left(k+M_{R}-1\right)$ are also available. Since $r\left(i+k+M_{R}\right)=r\left(k+M_{R}\right), \quad i=1,2, \ldots, \Delta r\left(k+M_{R}\right) \equiv 0$, the following system about preview information can be established:

$$
X_{R}(k+1)=A_{R} X_{R}(k),
$$

where

$$
\begin{aligned}
& X_{R}(k)=\left[\begin{array}{c}
\Delta r(k) \\
\Delta r(k+1) \\
\vdots \\
\Delta r\left(k+M_{R}-1\right)
\end{array}\right] \text {, } \\
& A_{R}=\left[\begin{array}{cccc}
0 & I_{q} & & \\
& 0 & \ddots & \\
& & \ddots & I_{q} \\
& & & 0
\end{array}\right] \text {. }
\end{aligned}
$$

Based on systems (11) and (13), the secondary augmented error system of subsystem (2) is

$$
\bar{x}(k+1)=\bar{A}_{i} \bar{x}(k)+\bar{B}_{i} \Delta u(k),
$$

where 


$$
\begin{aligned}
\bar{x}(k) & =\left[\begin{array}{c}
\widetilde{x}(k) \\
X_{R}(k)
\end{array}\right], \\
\bar{A}_{i} & =\left[\begin{array}{cc}
\widetilde{A}_{i} & F_{e} \\
0 & A_{R}
\end{array}\right], \\
\bar{B}_{i} & =\left[\begin{array}{c}
\widetilde{B}_{i} \\
0
\end{array}\right], \\
F_{e} & =\left[\begin{array}{llll}
B_{R} & 0 & \cdots & 0
\end{array}\right] .
\end{aligned}
$$

Corresponding to system (15), quadratic performance index function (6) can be rewritten as

$$
J=\sum_{k=k_{i}}^{\infty}\left[\lambda^{-2 k}\left(\bar{x}^{T}(k) \bar{Q} \bar{x}(k)+\Delta u^{T}(k) R \Delta u(k)\right)\right],
$$

where $k_{i}$ is the initial time of system (15) and $\bar{Q}=\operatorname{diag}\left\{Q_{e}, 0,0\right\}$.

For system (15), using the linear quadratic optimal control theory, it will be seen that $\Delta u(k)$ is designed to minimize quadratic performance index function (17), and correspondingly, the closed-loop system of system (15) is exponentially stable. Furthermore, if these stable closed-loop systems can be again proved to be switched stable, then it will illustrate that the error signal $e(k)$ can stabilize to zero exponentially. By analogy with the exponential stability of continuous-time linear systems in [23], the following lemma is obtained.

Lemma 1. Under assumptions $A 1$ and $A 2$, if $Q_{e}>0$, then the optimal input of system (15) that minimizes quadratic performance index function (17) is

$$
\Delta u(k)=F_{i} \bar{x}(k),
$$

which can make the closed-loop system of system (15) exponentially stable, that is,

$$
\|\bar{x}(k)\| \leq M_{i} \lambda^{k-k_{i}}\left\|\bar{x}\left(k_{i}\right)\right\|, \quad M_{i} \geq 1, i=1, \ldots, m .
$$

In (18),

$$
F_{i}=-\left[R+\left(\lambda^{-1} \bar{B}_{i}\right)^{T} \bar{P}_{i}\left(\lambda^{-1} \bar{B}_{i}\right)\right]^{-1}\left(\lambda^{-1} \bar{B}_{i}\right)^{T} \bar{P}_{i}\left(\lambda^{-1} \bar{A}_{i}\right)
$$

and $\bar{P}_{i}$ is the positive semidefinite solution of the algebraic Riccati equation

$$
\bar{P}_{i}=\bar{Q}+\left(\lambda^{-1} \bar{A}_{i}\right)^{T} \bar{P}_{i}\left(\lambda^{-1} \bar{A}_{i}\right)-\left(\lambda^{-1} \bar{A}_{i}\right)^{T} \bar{P}_{i}\left(\lambda^{-1} \bar{B}_{i}\right)\left[R+\left(\lambda^{-1} \bar{B}_{i}\right)^{T} \bar{P}_{i}\left(\lambda^{-1} \bar{B}_{i}\right)\right]^{-1}\left(\lambda^{-1} \bar{B}_{i}\right)^{T} \bar{P}_{i}\left(\lambda^{-1} \bar{A}_{i}\right)
$$

Proof. The proof has been divided into three steps:

Step 1: prove that the optimal controller with quadratic performance index function (17) is (18). By virtue of formula (17), the following transformation is needed:

$$
\left\{\begin{array}{l}
\widehat{x}(k)=\lambda^{-k} \bar{x}(k), \\
\Delta \widehat{u}(k)=\lambda^{-k} \Delta u(k),
\end{array}\right.
$$

which, combining with system (15), yields

$$
\widehat{x}(k+1)=\left(\lambda^{-1} \bar{A}_{i}\right) \widehat{x}(k)+\left(\lambda^{-1} \bar{B}_{i}\right) \Delta \widehat{u}(k) .
$$

Accordingly, formula (17) is rewritten as

$$
J=\sum_{k=k_{i}}^{\infty}\left[\left(\widehat{x}^{T}(k) \bar{Q} \widehat{x}(k)+\Delta \widehat{u}^{T}(k) R \Delta \widehat{u}(k)\right)\right] .
$$

According to Berkovitz [24], the optimal control of system (23) which minimizes quadratic performance index function (24) is

$$
\Delta \widehat{\mathcal{u}}(k)=F_{i} \widehat{x}(k) .
$$

This implies that the closed-loop system $\widehat{x}(k+1)=\left[\lambda^{-1}\left(\bar{A}_{i}+\bar{B}_{i} F_{i}\right)\right] \widehat{x}(k)$ is asymptotically stable, where $F_{i}$ is given in (20), and $\bar{P}_{i}$ satisfies algebraic Riccati equation (21). With (22) and (25), $\Delta u(k)=F_{i} \bar{x}(k)$ can be obtained.

Step 2: prove that equation (19) is valid. The asymptotical stability of the closed-loop system $\widehat{x}(k+1)=$ $\left[\lambda^{-1}\left(\bar{A}_{i}+\bar{B}_{i} F_{i}\right)\right] \widehat{x}(k)$ indicates that $\lim _{k \rightarrow \infty} \|\left[\lambda^{-1}\left(\bar{A}_{i}\right.\right.$ $\left.\left.+\bar{B}_{i} F_{i}\right)\right]^{k-k_{i}} \|=0$. Therefore, there exists a $K \geq k_{i}$ such that $\left\|\left[\lambda^{-1}\left(\bar{A}_{i}+\bar{B}_{i} F_{i}\right)\right]^{k-k_{i}}\right\|<1$ holds for any $k>K$. On this basis, $\max _{k \in\left\{k_{i}, k_{i}+1, \ldots, K\right\}}\left\|\left[\lambda^{-1}\left(\bar{A}_{i}+\bar{B}_{i} F_{i}\right)\right]^{k-k_{i}}\right\|=\bar{M}_{i}$ and $M_{i}=\max \left\{\bar{M}_{i}, 1\right\} \quad$ are defined. Then, $\left\|\left[\lambda^{-1}\left(\bar{A}_{i}+\bar{B}_{i} F_{i}\right)\right]^{k-k_{i}}\right\| \leq M_{i}\left(k \geq k_{i}\right) \quad$ is established. Therefore,

$$
\left\|\left(\bar{A}_{i}+\bar{B}_{i} F_{i}\right)^{k-k_{i}}\right\| \leq M_{i} \lambda^{k-k_{i}} .
$$

Note that the closed-loop system of (15) is $\bar{x}(k+1)=$ $\left(\bar{A}_{i}+\bar{B}_{i} F_{i}\right) \bar{x}(k)$. Then, $\bar{x}(k)=\left(\bar{A}_{i}+\bar{B}_{i} F_{i}\right)^{k-k_{i}} \bar{x}\left(k_{i}\right)$ could be obtained by iteration, and thus, $\|\bar{x}(k)\| \leq$

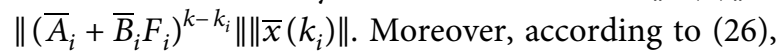
one has $\|\bar{x}(k)\| \leq M_{i} \lambda^{k-k_{i}}\left\|\bar{x}\left(k_{i}\right)\right\|$; therefore, (19) is derived.

Step 3: prove that $\bar{P}_{i}$ is a positive semidefinite solution of equation (21). It is noted that the detectability of $\left(C_{i}, A_{i}\right)$ and $Q_{e}>0$ indicate that $\left(\bar{Q}^{1 / 2}, \bar{A}_{i}\right)$ is detectable. 
Hence, the conclusion is obvious according to Liao et al. [25]. The proof is completed.

Based on Lemma 1, the preview control problem of switched system (4) is eventually transformed into the switched stability problem of the augmented error

$$
\begin{array}{r}
\bar{x}(k+1)=\bar{A}_{i} \bar{x}(k)+\bar{B}_{i} \Delta u(k), \quad k \in\left[k_{i}+l T, k_{i+1}+l T\right), \\
i=1, \ldots, m, l=0,1, \ldots,
\end{array}
$$

under controller (18).

\section{Switched Stability Analysis}

By Lemma 1, when subsystem (2) is the same, the closedloop system of system (27) can achieve asymptotic stability without considering the switching rule $\delta(k)$. However, when the subsystem is heterogeneous, the switching action can be viewed as the external disturbance for system (27), which may cause the unstability of the closed-loop system of system (27). The following conclusion will show the shortest working time (or dwell time $\tau$ [1]) required for the asymptotical stability of the whole switched system (27).

Theorem 1. Suppose that $A 1-A 3$ hold and $Q_{e}>0$. If the control input is (18), then the closed-loop system of system (27) is globally asymptotically stable for the periodic switching signal with the dwell time

$$
\tau>\tau^{*}=\max \left\{K, \frac{-\left(\sum_{j=1}^{m} \ln M_{j}\right)}{(m \ln \lambda)}\right\} .
$$

Proof. If the control input is (18), then the closed-loop system of system (27) is

$$
\bar{x}(k+1)=\left(\bar{A}_{i}+\bar{B}_{i} F_{i}\right) \bar{x}(k),
$$

where $k \in\left[l T+k_{i}, l T+k_{i+1}\right), i=1, \ldots, m$ and $l=0,1, \ldots$

Given the switching sequence, when $k \in\left[k_{1}, k_{2}\right)$, the solution of system (29) is

$$
\bar{x}(k)=\left(\bar{A}_{1}+\bar{B}_{1} F_{1}\right)^{k} \bar{x}(0),
$$

and for $k \in\left[k_{2}, k_{3}\right)$, the solution is

$$
\bar{x}(k)=\left(\bar{A}_{2}+\bar{B}_{2} F_{2}\right)^{\left(k-k_{2}\right)}\left(\bar{A}_{1}+\bar{B}_{1} F_{1}\right)^{T_{1}} \bar{x}(0) .
$$

Calculating system (27) recursively for any $k \in\left[k_{i}+l T, k_{i+1}+l T\right) \quad$ yields $\quad \bar{x}(k)=\left(\bar{A}_{i}+\bar{B}_{i} F_{i}\right)^{k-}$ $k_{i}-l T\left(\bar{A}_{i+1}+\bar{B}_{i-1} F_{i-} \quad 1\right)^{T_{i-1}} \ldots\left(\bar{A}_{1}+\bar{B}_{1} F_{1}\right)^{T_{1}}\left(\left(\bar{A}_{m}+\right.\right.$ $\left.\left.\bar{B}_{m} F_{m}\right)^{T_{m}} \cdots\left(\bar{A}_{1}+\bar{B}_{1} F_{1}\right)^{T_{1}}\right)^{l} \bar{x}(0)$. Taking the norm for the above equation gives

$$
\|\bar{x}(k)\| \leq\left\|\left(\bar{A}_{i}+\bar{B}_{i} F_{i}\right)^{k-k_{i}-l T}\right\|, \ldots,\left\|\left(\bar{A}_{1}+\bar{B}_{1} F_{1}\right)^{T_{1}}\right\|\left(\left\|\left(\bar{A}_{m}+\bar{B}_{m} F_{m}\right)^{T_{m}}\right\|, \ldots,\left\|\left(\bar{A}_{1}+\bar{B}_{1} F_{1}\right)^{T_{1}}\right\|\right)^{l}\|\bar{x}(0)\| .
$$

Utilizing conditions (19) and (28), the above equation can be estimated as

$$
\|\bar{x}(k)\| \leq M_{i} \lambda^{\left(k-k_{i-1}-l T\right)} M_{i-1} \lambda^{T_{i-1}}, \ldots, M_{1} \lambda^{T_{1}}\left(\Pi_{j=1}^{m} M_{j} \lambda^{T_{j}}\right)^{l}\|\bar{x}(0)\| .
$$

Denote $\quad \bar{L}=M_{i} \lambda^{k-k_{i-1}-l T} M_{i-1} \lambda^{T_{i-1}}, \ldots, M_{1} \lambda^{T_{1}} \quad$ and $\bar{L}=\prod_{j=1}^{m} M_{j}$; then, it follows from the value range of $k$ and $0<\lambda<1$ that $\bar{L}<L$, and thus,

$$
\|\bar{x}(k)\| \leq L\left(\prod_{j=1}^{m} M_{j} \lambda^{T_{j}}\right)^{l}\|\bar{x}(0)\| .
$$

By $T_{i} \geq \tau$, it can be easily obtained that

$$
\|\bar{x}(k)\| \leq L \gamma^{l}\|\bar{x}(0)\|,
$$

where $\gamma=\prod_{j=1}^{m} M_{j} \lambda^{\tau}$.

Note that $\gamma=\prod_{j=1}^{m} M_{j} \lambda^{\tau}=\exp \left(\tau \ln \lambda+\sum_{j=1}^{m} \ln M_{j}\right)$, according to $0<\lambda<1$ and (28), and simple calculation gives that $0<\gamma<1$. Because of $k \in\left[l T+k_{i}, l T+k_{i+1}\right)$, taking the limit of (35) yields $\lim _{k \rightarrow \infty}\|\bar{x}(k)\| \leq \lim _{l \rightarrow \infty} L \gamma^{l}\|\bar{x}(0)\|=0$, which means that the zero solution of system (29) is globally asymptotically stable. The proof is completed.

\section{Design of the Optimal Preview Controller}

It follows from Theorem 1 that switched closed-loop augmented system (29) is global asymptotically stable under assumptions A1-A3 and the dwell-time condition (28). Therefore, the state component $e(k)$ will converge to zero as time tends to infinity with the optimal control input (18) in Theorem 1, which means that the switched preview tracking problems can be solved by controller (18). According to Theorem 1 and the above discussion, the following result provides the sufficient conditions and the specific form of the preview controller for realizing the switched preview tracking control for system (4).

Theorem 2. Under the periodic switching sequence, if

(i) $Q_{e}>0$ and $R>0$,

(ii) Assumptions A1-A3 hold, and

(iii) The dwell time $\tau>\tau^{*}=\max \left\{K,-\left(\sum_{j=1}^{m} \ln M_{j}\right) /\right.$ $(m \ln \lambda)\}$,

then the optimal preview controller for achieving the preview tracking of system (4) is 


$$
u(k)=F_{e i} \sum_{l=0}^{k-1} e(l-1)+F_{x i} x(k)+\sum_{j=0}^{M_{R}-1} F_{R i}(j)(r(k+j)-r(j)),
$$

where

$$
\begin{aligned}
& k \in\left(k_{i}+l T, k_{i+1}+l T\right], i=1, \ldots, m, \\
& F_{0 i}=\left[\begin{array}{ll}
F_{e i} & F_{x i}
\end{array}\right]=-\left[R+\left(\lambda^{-1} \widetilde{B}_{i}\right)^{T} P_{i}\left(\lambda^{-1} \widetilde{B}_{i}\right)\right]^{-1}\left(\lambda^{-1} \widetilde{B}_{i}\right)^{T} P_{i}\left(\lambda^{-1} \widetilde{A}_{i}\right) \text {, } \\
& F_{R i}(0)=-\left(R_{i}+\left(\lambda^{-1} \widetilde{B}_{i}\right)^{T} P_{i}\left(\lambda^{-1} \widetilde{B}_{i}\right)\right)^{-1}\left(\lambda^{-1} \widetilde{B}_{i}\right)^{T} P_{i}\left(\lambda^{-1} B_{R}\right), \\
& F_{R i}(j)=-\left(R_{i}+\left(\lambda^{-1} \widetilde{B}_{i}\right)^{T} P_{i}\left(\lambda^{-1} \widetilde{B}_{i}\right)\right)^{-1}\left(\lambda^{-1} \widetilde{B}_{i}\right)^{T}\left(\xi_{i}^{T}\right)^{j} P_{i}\left(\lambda^{-1} B_{R}\right) \\
& \xi_{i}=\left(\lambda^{-1} \widetilde{A}_{i}\right)+\left(\lambda^{-1} \widetilde{B}_{i}\right) F_{0 i}, \quad j=1, \ldots, M_{R}-1,
\end{aligned}
$$

and $P_{i}$ is the solution of the following reduced-order algebraic Riccati equation:

$$
\begin{aligned}
P_{i}= & Q+\left(\lambda^{-1} \widetilde{A}_{i}\right)^{T} P_{i}\left(\lambda^{-1} \widetilde{A}_{i}\right)-\left(\lambda^{-1} \widetilde{A}_{i}\right)^{T} P_{i}\left(\lambda^{-1} \widetilde{B}_{i}\right) \\
& \cdot\left[R+\left(\lambda^{-1} \widetilde{B}_{i}\right)^{T} P_{i}\left(\lambda^{-1} \widetilde{B}_{i}\right)\right]^{-1}\left(\lambda^{-1} \widetilde{B}_{i}\right)^{T} P_{i}\left(\lambda^{-1} \widetilde{A}_{i}\right) .
\end{aligned}
$$

Proof. Under conditions (i)-(iii), the conclusion of Theorem 1 holds, which implies that error vector $e(k)$ will decay to zero asymptotically, that is, the preview tracking of system (4) can be realized with the control input (18).

Let

$$
\begin{aligned}
\bar{P}_{i} & =\left[\begin{array}{ll}
P_{i} & S \\
S^{T} & Z
\end{array}\right], \\
F_{i} & =\left[\begin{array}{lllll}
F_{e i} & F_{x i} & F_{R i}(0) & \cdots & F_{R i}\left(M_{R}-1\right)
\end{array}\right] .
\end{aligned}
$$

Then, the results (37)-(41) can be obtained by applying the method in [26] to equation (21). The specific derivation process is omitted here. Interested readers can refer to [26].

For the optimal preview control (36), according to (37)-(40), formula (18) can be expressed as the following form:

$$
\begin{aligned}
\Delta u(k) & =F_{0 i} \tilde{x}(k)+\sum_{j=0}^{M_{R}-1} F_{R i}(j) \Delta r(k+j) \\
& =F_{e i} e(k)+F_{x i} \Delta x(k)+\sum_{j=0}^{M_{R}-1} F_{R i}(j) \Delta r(k+j) .
\end{aligned}
$$

By the definition of difference operator $\Delta v(k)=v(k+1)-v(k),(43)$ can be reformulated as

$$
\begin{aligned}
u(k+1)-u(k)= & F_{e i} e(k)+F_{x i}(x(k+1)-x(k)) \\
& +\sum_{j=0}^{M_{R}-1} F_{R i}(j)(r(k+j+1)-r(k+j)) .
\end{aligned}
$$

Furthermore, shifting $u(k)$ to the right side of the above equation gives

$$
\begin{aligned}
u(k+1)= & u(k)+F_{e i} e(k)+F_{x i}(x(k+1)-x(k)) \\
& +\sum_{j=0}^{M_{R}-1} F_{R i}(j)(r(k+j+1)-r(k+j)) .
\end{aligned}
$$

Replacing $k$ with $k-1$, there exists

$$
\begin{aligned}
u(k)= & u(k-1)+F_{e i} e(k-1)+F_{x i}(x(k)-x(k-1)) \\
& +\sum_{j=0}^{M_{R}-1} F_{R i}(j)(r(k+j)-r(k+j-1)) .
\end{aligned}
$$

Finally, let $u(k)=0, x(k)=0$, and $r(k)=0$ hold for $k \leq 0$; then, the optimal preview controller (36) can be obtained from the last equation by the recursive way. The proof is completed.

Remark 3. In controller (36), $F_{e i} \sum_{l=0}^{k-1} e(l-1)$ is the error integral term and can eliminate the steady-state error in the tracking process; $F_{x i} x(k)$ denotes the state feedback; and $\sum_{j=0}^{M_{R}-1} F_{R i}(j)(r(k+j)-r(j))$ represents the preview feedforward compensation and can improve the tracking performance, such as tracking accuracy, tracking speed, and adjusting time. The structure of the switched preview tracking control of system (1) under controller (36) is shown in Figure 1.

Remark 4. In fact, the periodically switched systems studied in this paper can also be regarded as a periodic system. The results about preview tracking control of the periodic system have been reported in [22]. The core idea of [22] is that the lifting method is applied to convert the original linear periodic system into a time-invariant system. This procedure can lead to a limitation since the stabilizability of the original periodic system has no relationship with the stabilizability of the time-invariant system, which will increase the complexity of the system design. However, after constructing the augmented error system for each subsystem in this paper, the remaining is to search the dwell-time condition for guaranteeing the switched stability of the whole closed-loop augmented error subsystem.

Remark 5. Note that this paper only studies the switched preview tracking problem based on the dwell-time approach. However, if the switching signals are modeled by a Markov chain, then the problem will become complex and challenging. So far, many useful results have been reported [27-29]. In [27], $H_{\infty}$ estimation problem was concerned 


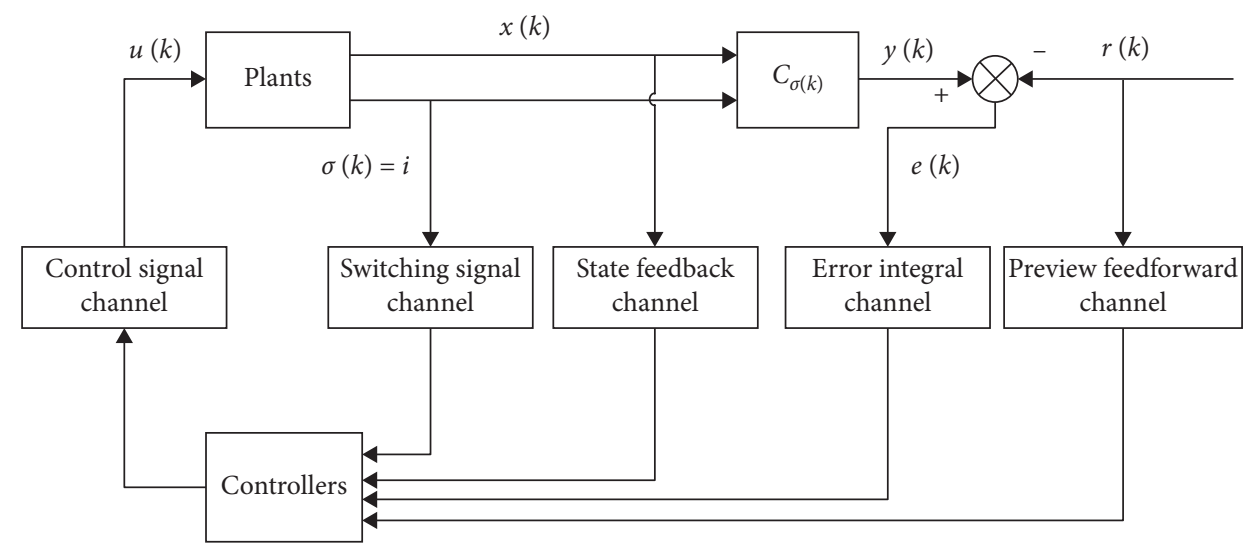

FIGURE 1: Block diagram of the switched preview tracking control system.

for the discrete-time Markov jump system. Liu et al. [28] considered an asynchronous $H_{\infty}$ control problem for networked Markovian jump systems. Liu et al. [29] investigated the quasi-synchronous control problem of continuous-time heterogeneous networks with a generalized Markovian topology. Inspired by the literature aforementioned, future work will be focused on the switched preview tracking problem for linear discrete-time Markovian jump systems based on the stochastic Lyapunov stable theory.

Remark 6. This paper considers the situation that the switching signals of the systems and the controller are synchronous. However, if they are asynchronous, then the proposed method in this paper cannot be directly generalized to tackle the corresponding switching preview tracking problem since the dwell-time conditions required for the asymptotic stability are different for the two classes of systems. Therefore, the preview control problem of switched systems under asynchronous switching will be an interesting and challenging research topic.

\section{Numerical Simulation}

Consider a discrete-time linear switched system with the form of (4), where the coefficient matrices are

$$
\begin{aligned}
A_{1} & =\left[\begin{array}{ll}
0.5 & 0.2 \\
0.5 & 1
\end{array}\right], \\
B_{1} & =\left[\begin{array}{c}
0.3 \\
1
\end{array}\right], \\
C_{1} & =\left[\begin{array}{ll}
0.7 & 0.5
\end{array}\right], \\
A_{2} & =\left[\begin{array}{ll}
0.3 & 1 \\
1 & 0.2
\end{array}\right], \\
B_{2} & =\left[\begin{array}{l}
1.2 \\
1
\end{array}\right], \\
C_{2} & =\left[\begin{array}{ll}
0.5 & 0.6
\end{array}\right] .
\end{aligned}
$$

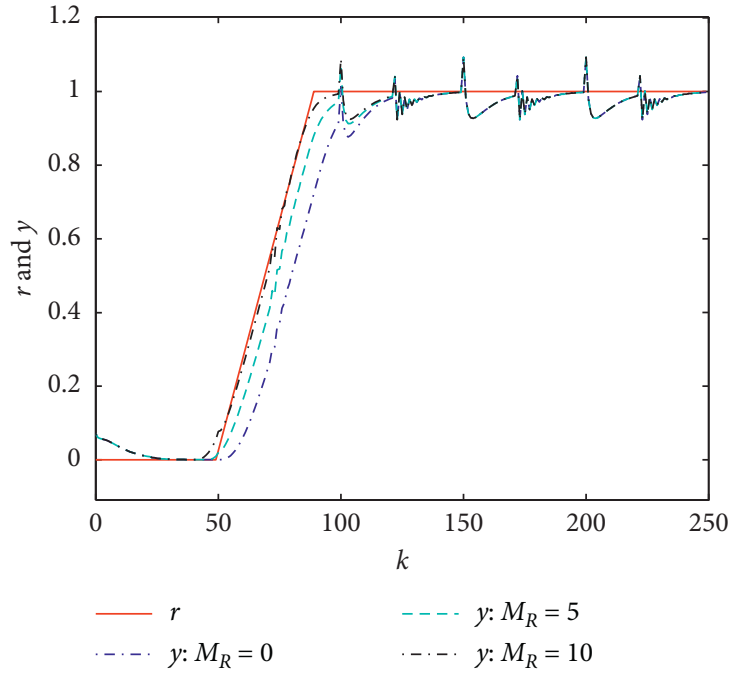

Figure 2: The output responses of system (4) under different preview lengths $\left(T_{1}=22\right.$ and $\left.T_{2}=28\right)$.

$$
\text { Choose } x(0)=\left[\begin{array}{c}
0.1 \\
0
\end{array}\right], \quad u(0)=0, Q_{e}=0.0001 \text {, and }
$$

$R=1$. In addition, let $\lambda=0.94$ and $K=21$. It can be easily obtained that $M_{1}=2.23$ and $M_{2}=1.85$. Substituting them into (28) gives $\tau>\max \{21,11.45\}=21$. Based on this result, $T_{1}=22$ and $T_{2}=28$ are taken in the following simulation.

Moreover, it can be verified that system (4) satisfies assumptions 1 and 2. By solving Riccati equation (41), $F_{e 1}=-0.04068, F_{x 1}=[-0.33332-0.40959], F_{e 2}=-0.03073$, and $F_{x 2}=[-0.31065-0.29769]$ are obtained.

The reference signal $r(k)$ is taken as

$$
r(k)= \begin{cases}0, & 0 \leq k \leq 50 \\ 0.025(k-50), & 50<k \leq 90 \\ 1, & k>90 .\end{cases}
$$

It is assumed that $r(k)$ is previewable. In the following, the simulations will be performed in three cases, that is, $M_{R}=0, M_{R}=5$, and $M_{R}=10$.

The output response curves under different preview lengths are shown in Figure 2. 


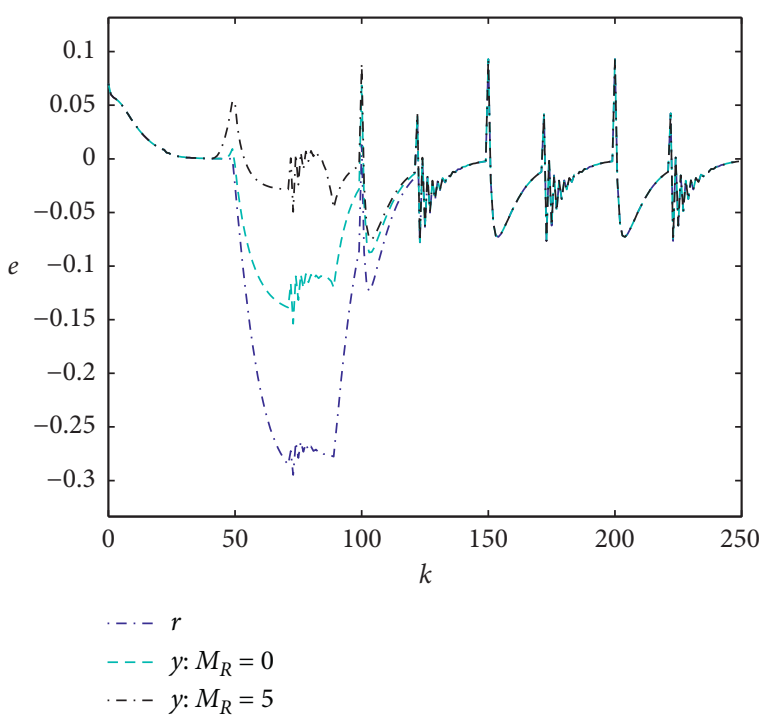

FIGURE 3: The tracking error curves under different preview lengths $\left(T_{1}=22\right.$ and $\left.T_{2}=28\right)$.

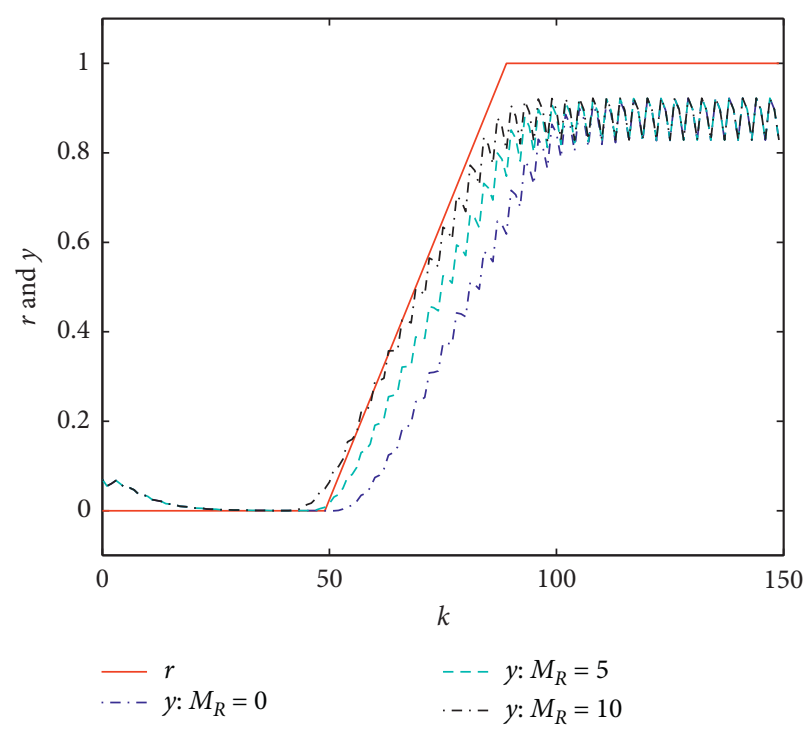

Figure 4: The output responses of system (4) under different preview lengths $\left(T_{1}=1\right.$ and $\left.T_{2}=2\right)$.

It can be observed from Figure 2 that the switched system can track the reference signal $r(k)$. Moreover, the output can track the reference signal more precisely with the increase of the preview length. In addition, it is necessary to point out that the time when the jump occurs in the figure is exactly the time when the system switches.

In Figure 3, it can be found that the error signal can tend to zero during each running period $T_{i}=k_{i+1}-k_{i}$. Moreover, when switching occurs among the subsystems, the error signal fluctuates at the switching point. However, with the increase of the preview length, it can be seen that the error signal decreases in a certain range.

In order to verify the importance of dwell-time condition (28) in Theorem 2, the running time of the subsystems will be taken as $T_{1}=1$ and $T_{2}=2$ (both are less than the dwell time), respectively. The reference signal $r(k)$ and preview lengths are unchanged, and the simulation results are shown in Figure 4.

From Figure 4, we can clearly see that the output does not track the reference signal. Owing to the shortage of the running time, the output has to switch before reaching the reference signal's maximum amplitude. Therefore, the dwell time of the system is very important to ensure the tracking performance.

\section{Conclusion}

In this paper, the preview tracking problem of the periodic switched systems has been investigated. The augmented error system method is adopted to complete the reformulation of the original problem. The switched system stability theory is used to provide the sufficient conditions for achieving the asymptotic preview tracking control. The gain matrices of the optimal preview control are designed from the perspective of a linear quadratic optimal method.

\section{Data Availability}

No data were used to support this study.

\section{Conflicts of Interest}

The authors declare that there are no conflicts of interest.

\section{Acknowledgments}

This work was supported by the Fundamental Research Funds for the Central Universities (FRF-TP-18-101A1).

\section{References}

[1] D. Liberzon, Switching in Systems and Control, Birkhäuser, Boston, MA, USA, 2003.

[2] J. Zhao and G. M. Dimirovski, "Quadratic stability of a class of switched nonlinear systems," IEEE Transactions on Automatic Control, vol. 49, no. 4, pp. 574-578, 2004.

[3] M. Margaliot, "Stability analysis of switched systems using variational principles: an introduction," Automatica, vol. 42, no. 12, pp. 2059-2077, 2006.

[4] H. Lin and P. J. Antsaklis, "Stability and stabilizability of switched linear systems: a survey of recent results," IEEE Transactions on Automatic Control, vol. 54, no. 2, pp. 308322, 2009.

[5] Y. Mo and D. Xiao, "Overview of hybrid dynamic system and its application," Control Theory \& Applications, vol. 19, no. 1, pp. 1-8, 2002.

[6] Y. Wu and G. Zong, "Relay switching controller with finite time tracking for rigid robotic manipulators," Acta Automatica Sinica, vol. 31, no. 3, pp. 412-418, 2005.

[7] A. Sargolzaei, K. K. Yen, M. N. Abdelghani, S. Sargolzaei, and B. Carbunar, "Resilient design of networked control systems under time delay switch attacks, application in smart grid," IEEE Access, vol. 5, pp. 15901-15912, 2017.

[8] A. Grzech, "Flow control in virtual circuit-oriented packetswitched computer communication network," Annual Review in Automatic Programming, vol. 12, no. P1, pp. 437-440, 1985. 
[9] J. Lu and G. Zhao, "Stability analysis of discrete time-delay switched systems," Information and Control, vol. 34, no. 3, pp. 381-384, 2005.

[10] W. Sun, S. Dong, and K. Zou, "Asymptotic stability of linear periodic switched systems," Journal of University of Science and Technology Liaoning, vol. 25, no. 1, pp. 158-160, 2006.

[11] J. Hespanha, "Uniform stability of switched linear systems: extensions of LaSalle's Invariance Principle," IEEE Transactions on Automatic Control, vol. 49, no. 4, pp. 470-482, 2004.

[12] X. Zhao, L. Zhang, P. Shi, and M. Liu, "Stability and stabilization of switched linear systems with mode-dependent average dwell time," IEEE Transactions on Automatic Control, vol. 57, no. 7, pp. 1809-1815, 2012.

[13] J.-L. Wu, "Feedback stabilization for multiinput switched nonlinear systems: two subsystems case," IEEE Transactions on Automatic Control, vol. 53, no. 4, pp. 1037-1042, 2008.

[14] S. Li, J. Guo, and Z. Xiang, "Global stabilization of a class of switched nonlinear systems under sampled-data control," IEEE Transactions on Systems, Man, and Cybernetics: Systems, vol. 49, no. 9, pp. 1912-1919, 2019.

[15] S. Li, C. K. Ahn, and Z. Xiang, "Sampled-data adaptive output feedback fuzzy stabilization for switched nonlinear systems with asynchronous switching," IEEE Transactions on Fuzzy Systems, vol. 27, no. 1, pp. 200-205, 2019.

[16] T. Katayama, T. Ohki, T. Inoue, and T. Kato, "Design of an optimal controller for a discrete-time system subject to previewable demand," International Journal of Control, vol. 41, no. 3, pp. 677-699, 1985.

[17] M. Cao and F. Liao, "Design of an optimal preview controller for linear discrete-time descriptor noncausal multirate systems," The Scientific World Journal, vol. 2014, Article ID 965915, 11 pages, 2014.

[18] L. Li and F. Liao, "Robust preview control for a class of uncertain discrete-time systems with time-varying delay," ISA Transactions, vol. 73, pp. 11-21, 2018.

[19] L. Li and Y. Yuan, "Output feedback preview control for polytopic uncertain discrete-time systems with time-varying delay," International Journal of Robust and Nonlinear Control, vol. 29, no. 9, pp. 2619-2638, 2019.

[20] H. Wang, M. Fu, and H. Zhang, "Ho control for stochastic systems with disturbance preview," SIAM Journal on Control and Optimization, vol. 58, no. 1, pp. 215-242, 2020.

[21] H. Yoshiro, "New LMI-based conditions for preview feedforward synthesis," Control Engineering Practice, vol. 90, pp. 19-26, 2019.

[22] F. Liao, M. Sun, and Usman, "Optimal preview control for linear discrete-time periodic systems," Mathematical Problems in Engineering, vol. 2019, Article ID 8434293, 11 pages, 2019.

[23] B. D. O. Anderson and J. B. Moore, Optimal Control: Linear Quadratic Method, Prentice-Hall, Englewood Cliffs, NJ, USA, 1990.

[24] L. D. Berkovitz, Optimal Control Theory, Springer-Verlag, Beijing, China, 1974.

[25] F. Liao, K. Takaba, T. Katayama, and J. Katsuura, "Design of an optimal preview servomechanism for discrete-time systems in a multirate setting," Dynamics of Continuous, Discrete and Impulsive Systems, vol. 10, no. 5, pp. 727-744, 2003.

[26] T. Takeshi and E. Tadashi, Digital Preview and Predictive Control, Beijing Science and Technology Press, Beijing, China, 1994.

[27] L. Zhang, "Ho estimation for discrete-time piecewise homogeneous Markov jump linear systems," Automatica, vol. 45, pp. 2570-2576, 2009.
[28] X. Liu, G. Ma, P. R. Pagilla, and S. S. Ge, "Dynamic output feedback asynchronous control of networked Markovian jump systems," IEEE Transactions on Systems, Man, and Cybernetics: Systems, vol. 50, no. 7, pp. 2705-2715, 2020.

[29] X. Liu, W. P. Tay, Z. Liu, and G. Xiao, "Quasi-synchronization of heterogeneous networks with a generalized Markovian topology and event-triggered communication," IEEE Transactions on Cybernetics, vol. 50, no. 10, pp. 4200-4213, 2019. 